\title{
Insulin Pump Therapy - Influence on Body Fat Redistribution, Skeletal Muscle Mass and Ghrelin, Leptin Changes in T1D Patients
}

\author{
Dana Prídavkováa Matej Samoša Ivana Kazimierováb ${ }^{\circ} \quad$ Ĺudovít Šutarík $^{a}$ \\ Soňa Fraňováb Peter Galajda ${ }^{a}$ Marián Mokáň ${ }^{a}$ \\ ${ }^{a}$ Clinic of Internal Medicine, Jessenius Faculty of Medicine in Martin (JFM CU), Comenius \\ University in Bratislava, Bratislava, Slovakia; ${ }^{b}$ 1Biomedical Centre Martin JFM CU and \\ 2Department of Pharmacology JFM CU, Jessenius Faculty of Medicine in Martin (JFM CU), \\ Comenius University in Bratislava, Bratislava, Slovakia
}

\section{Keywords}

Insulin pump therapy · Type 1 diabetes mellitus · Visceral fat · Leptin · Ghrelin

\section{Abstract}

Background: To report changes in body composition and biochemical parameters in patients with type 1 diabetes mellitus (T1D) after switching from multiple daily injection (MDI) to continuous subcutaneous insulin infusion (CSII). Methods: 31 patients switched over from MDI to CSII. Body composition, biochemical parameters, glycaemic variability (GV) and level of physical activity were evaluated before and 6 months on CSII. Results: In both sexes, we found an increase in skeletal muscle mass (SMM), $(p=0.008 ; 0.008)$. In men, there was mainly a decrease in visceral fat area (VFA), $(p=0.028)$ and in women there was decrease of total body fat (TBF), $(p=0.020)$ and non-significant decrease of VFA $(p=0.098)$. SMM inversely correlated with VFA in men $(p=-0.001)$ and with TBF in women $(p=-0.005)$. GV was decreased generally and correlated inversely with TBF in men only $(p=-0.026)$. Physical activity was increased and correlated inversely with VFA in men $(p=-0.002)$ and in women $(p=-0.006)$. Conclusions: Using CSII in T1D leads to a significant increase of SMM in both sexes to a decrease of VFA in men and to a non-significant decrease of VFA in women. Changes in adipose tissue and SMM were also related to increased physical activity and to decreased GV. 
Prídavková et al.: Insulin Pump Therapy - Influence on Body Fat Redistribution, Skeletal Muscle Mass and Ghrelin, Leptin Changes in T1D Patients

\section{Introduction}

Recent advances have revealed new information about the distribution and biological characteristics of adipose tissue. Fewer studies have been completed on type 1 diabetes mellitus (T1D) patients. However, there is growing evidence about the association of the parameters of metabolic syndrome (MS) and T1D, known as 'double diabetes' - where abdominal obesity plays the main role. The reported prevalence of MS in T1D is rather high $-30-45 \%$ [1] in adults and about 3.2\% in children with T1D, with one-third of the children being overweight or obese [2].

In general, the diabetic population has higher visceral and intermuscular fat and lower subcutaneous fat [3]. In non-obese women with T1D, the higher central depots of fats are the main determinant of MS, while peripheral fat distribution and total fat percentage show no correlation with MS [1]. Men without diabetes have higher visceral adiposity compared to women without diabetes, while men with diabetes have lower visceral adiposity compared to women with diabetes [4]. Results of The Diabetes Control and Complications Trial Research Group (DCCT) showed that intensive glycaemic control reduces the incidence and progression of microvascular complications in T1D [5], but also leads to body weight (BW) increase [6]. There are few studies with adult patients with T1D, which have focused on fat distribution changes after switching from multiple daily injection (MDI) to continuous subcutaneous insulin infusion (CSII). Jacob et al. [7] found that an increase in BW in T1D patients was mostly based on the intrinsic lipogenic effect of insulin, which leads to an increase of fat and lean mass. Lipsky et al. [8] presented similar results - the lipogenic effect of insulin may be the primary cause of increased BW, but the frequency of hypoglycaemic episodes and appetite score does not correlate with BW increase. Excess weight gain was associated also with lower bolus-to-basal-insulin ratio independent of glycaemic control and activity level [9]. Other authors associated excess weight gain with increase of the visceral-to-subcutaneous-fat ratio; the changes in this ratio negatively correlated with physical activity, but not with HbA1c changes [10]. There are not many proofs comparing changes of body composition in patients with T1D after switching from MDI to CSII. DCCT and other studies are, among other factors, focused on changes of BW, body composition on intensified regimen or compared the prevalence of metabolic syndrome on CSII $[1,7,10,11]$.

We aimed to find out, how CSII does influence body composition and biochemical changes, e.g. leptin and ghrelin, and how the level of physical activity and glycaemic variability (GV) does change. Given the prospective character of the study and its easy reproducibility, we used bioelectrical impedance in a similar way as in the DCCT study when evaluating the changes in body composition [11]. We postulated that an improvement of GV would lead to a decline in hypoglycaemic episode frequency and thus to a reduction of carbohydrate intake as well as an improvement of insulin sensitivity with the added benefit of an anticatabolic effect of insulin.

\section{Subjects and Methods}

We examined 31 T1D patients (15 men, 16 women) admitted while being switched from MDI to CSII. $24 \%$ of men but none of the women were smokers, $10 \%$ had microalbuminuria, $8 \%$ retinopathy and $11 \%$ took angiotensin-converting enzyme inhibitor (ACE-i) due to nephrological indications or for arterial hypertension. Subjects with co-morbidities or oedemas and pregnant patients were excluded. Anthropometric and biochemical measurements were taken before being started on pump therapy and 6 months after initiation of therapy. The examinations were identical, while fasting, before noon, and without significant physical activity. BMI is defined as weight in kilograms divided by the square of the height in metres $\left(\mathrm{kg} / \mathrm{m}^{2}\right)$; the normal range was $18.5-24.9 \mathrm{~kg} / \mathrm{m}^{2}$. Waist circumference (WC) was measured at the midpoint between the 
Prídavková et al.: Insulin Pump Therapy - Influence on Body Fat Redistribution, Skeletal Muscle Mass and Ghrelin, Leptin Changes in T1D Patients

lower margin of the rib cage and the upper margin of the iliac crest as reported by the WHO (World Health Organization) in cm [12]. Cut-off points for WC were $94 \mathrm{~cm}$ for men and $80 \mathrm{~cm}$ for women as determined for a European population [13].

Neck circumference (NC), as an anthropometric parameter of 'upper body obesity', was measured using an elastic tape in a standardised manner horizontally below the cricothyroid cartilage at the level of the mid cervical spine to $1 \mathrm{~mm}$ accuracy [14]. Measurements of total body fat (TBF) in \%, visceral fat area (VFA) in $\mathrm{cm}^{2}$ and skeletal muscle mass (SMM) in kg were taken via a bioelectrical impedance analysis (BIA) device (InBodyS10, InBody Co., Ltd., Seoul, South Korea) using an eight-point tactile electrode system with 30 impedance measurements and six frequencies $(1,5,50,250,500,1,000 \mathrm{kHz})$ at each of the five segments (right and left arm, trunk, right and left leg) in a sitting position. Skinfold thickness was measured on the right side of the body by Harpenden caliper (HSB-IB; Baty International, Burgess Hill, UK), with constant closing compression of 10 g. $\mathrm{mm}^{2}$ throughout the range of measurements, with calibration to at least $40 \mathrm{~mm}$ with an accuracy of $0.2 \mathrm{~mm}$. The following skinfolds (in $\mathrm{cm}$ ) were measured: triceps skinfold (TS), biceps skinfold (BS), subscapular skinfold (sSS) and suprailiac skinfold (SIS), and recorded to the nearest $0.1 \mathrm{~cm}$. We set indexes such as subscapular-to-triceps ratio (sS/T ratio) and sSS+SIS sum. Anthropometric measurements were taken according to ISAK standards (International Society for Advancement of Kinanthropometry) [15]. Glycaemic levels were determined by enzymatic UV test (hexokinase method) (OSR6221, BeckmanCoulter, AU680; Beckman Coulter, Brea, CA, USA) in mmol/L, levels of TC (total-cholesterol) were determined by enzymatic colour test (CH202, Randox, AU680; Beckman Coulter) in mmol/L; levels of HDL-C (high-density lipoprotein cholesterol) were determined by enzymatic clearance assay for the in vitro quantitative determination (CH 2655 Randox, analyser AU680, Beckman Coulter) in mmol/L; levels of LDL-C (low-density lipoprotein cholesterol) were calculated using Friedewald's equation: TC - HDL-C - (TAG/2.2) in mmol/L; levels of TAG (triglycerides) were determined by enzymatic colour test (OSR61118 Beckman Coulter, analyser AU680, Beckman Coulter) in mmol/L; levels of TP (total proteins) were determined by photometric colour test (OSR6221 Beckman Coulter, analyser AU680, Beckman Coulter) in g/L; and levels of HbA1c was determined by high-performance liquid chromatography, HPCL (D-10 ${ }^{\mathrm{TM}}$ haemoglobin A1c Reorder Pack, analyser; Bio-Rad D, Hercules, CA, USA) in $\mathrm{mmol} / \mathrm{mol}$. The standardisation program is traceable to the reference methods of both the National Glycohaemoglobin Standardization Program (NGSP) and the International Federation of Clinical Chemistry and Laboratory Medicine (IFCC). We set total daily dose of insulin (TDDI) per kg BW. The levels of ghrelin and leptin were quantified in serum by the apparatus Bio-Plex ${ }^{\circledR} 200$ System (kitBio-Plex Pro ${ }^{\mathrm{TM}}$ Diabetes Standard; (Bio-). Output data were assessed by Bio-Plex Manager ${ }^{\mathrm{TM}} 6.0$ software, and results were presented in $\mathrm{pg} / \mathrm{mL}$. Using self-monitored blood glucose (SMBG) from 14 days with 3-5 readings/day over 1 month, we calculated parameters of GV - average daily risk range (ADRR) according to Kovatchev et al. [16], with values $<20$ for low risk, 20-40 for moderate risk and $>40$ for high risk - and standard deviation (SD) according to Siegellar et al. [17] with normal range 0-3. Total energy expenditure (TEE) was evaluated using 7-Day Physical Activity Recall [18]. Metabolic equivalents (METs) were calculated using the compendium of physical activities, and total kilocalories of energy expenditure per day were calculated [19]. One MET was defined as energy expenditure while sitting quietly, which, for an average adult, approximates $3.5 \mathrm{~mL}$ of oxygen uptake per kilogram of BW per min [20].

\section{Statistical Analyses}

Data are presented as frequency ( $n$ ), as percentage (\%) for categorical variables and as mean \pm standard deviation (SD), 95\% confidence interval and median for continuous variables. All tests used were non-parametric. The Wilcoxon signed-rank test was used to compare the difference between the baseline and followup value of observed parameters. The Mann-Whitney U test was used to compare baseline continuous variables between independent groups. Correlations between observed parameters were examined using Spearman rank correlation (@). All tests were two-tailed, and significance was set to $\alpha=0.05$. Analysis was performed with the SPSS 23.0 software package (IBM, Armonk, NY, USA).

\section{Results}

In response to switching from multiple daily injections to continuous subcutaneous insulin infusion, VFA was significantly reduced in men $(p=0.028)$ and non-significantly in women $(p$ $=0,098)$. SMM was increased in men $(p=0.008)$ and in women $(p=0.008)$ (Tables 1,2 , 
Prídavková et al.: Insulin Pump Therapy - Influence on Body Fat Redistribution, Skeletal Muscle Mass and Ghrelin, Leptin Changes in T1D Patients

Table 1. Anthropometric, biochemical parameters, glycaemic variability, energy expenditure in men

\begin{tabular}{|c|c|c|c|c|c|c|c|c|}
\hline \multirow{2}{*}{$\begin{array}{l}\text { Men } \\
n=15\end{array}$} & \multicolumn{3}{|l|}{ Before pump } & \multicolumn{3}{|l|}{ On pump } & \multirow{2}{*}{$\begin{array}{l}\text { Mean } \\
\text { change }\end{array}$} & \multirow[t]{2}{*}{$p$ value } \\
\hline & $\mathrm{MV} \pm \mathrm{SD}$ & $95 \% \mathrm{CI}$ & $\begin{array}{l}\text { Med } \\
\text { (x) }\end{array}$ & $\mathrm{MV} \pm \mathrm{SD}$ & $95 \%$ CI & $\begin{array}{l}\text { Med } \\
\text { (x) }\end{array}$ & & \\
\hline \multicolumn{9}{|c|}{ Anthropometric parameters } \\
\hline BW & $83.3 \pm 12.6$ & 177,183 & 81.0 & $85.1 \pm 12.8$ & $78.0,92.2$ & 84.0 & 1.9 & $0.045^{*}$ \\
\hline BMI & $25.9 \pm 4.3$ & $23.5,28.2$ & 25.2 & $26.4 \pm 4.3$ & $24.0,28.8$ & 25.0 & 0.6 & 0.059 \\
\hline WC & $88.8 \pm 12.1$ & $82.1,95.6$ & 87.0 & $90.9 \pm 13.5$ & $83.4,98.4$ & 87.5 & 2.1 & 0.285 \\
\hline NC & $38.8 \pm 2.7$ & $37.3,40.3$ & 39.0 & $39.2 \pm 2.6$ & $37.8,40.6$ & 39.0 & 0.4 & $0.025^{*}$ \\
\hline \multicolumn{9}{|c|}{ Bioelectrical impedance analysis } \\
\hline TBF & $14.5 \pm 7.9$ & $10.1,18.9$ & 14.4 & $13.6 \pm 6.6$ & $9.9,17.2$ & 12.5 & -1.0 & 0.496 \\
\hline VFA & $44.8 \pm 34.2$ & $25.8,63.7$ & 36.4 & $36.2 \pm 29.3$ & $20.0,52.4$ & 33.4 & -8.5 & $0.028^{*}$ \\
\hline SMM & $40.2 \pm 4.2$ & $37.9,42.5$ & 39.6 & $41.9 \pm 4.7$ & $39.3,44.5$ & 42.0 & 1.7 & $0.008^{* *}$ \\
\hline \multicolumn{9}{|l|}{ Skinfold thickness } \\
\hline BS & $0.71 \pm 0.54$ & $0.41,1.01$ & 0.45 & $0.76 \pm 0.36$ & $0.55,0.96$ & 0.7 & 0.05 & 0.287 \\
\hline TS & $1.19 \pm 0.52$ & $0.91,1.48$ & 1.1 & $1.28 \pm 0.49$ & $1.01,1.55$ & 1.2 & 0.09 & 0.637 \\
\hline sSS & $1.91 \pm 0.93$ & $1.4,2.43$ & 2.0 & $1.90 \pm 0.93$ & $1.38,2.42$ & 1.8 & -0.01 & 1.0 \\
\hline SIS & $1.57 \pm 0.66$ & $1.21,1.94$ & 1.7 & $1.94 \pm 0.72$ & $1.54,2.34$ & 2.0 & 0.37 & $0.004^{* *}$ \\
\hline sSS+SIS & $3.49 \pm 1.52$ & $2.64,4.33$ & 3.6 & $3.84 \pm 1.55$ & $2.98,4.70$ & 3.6 & 0.35 & 0.068 \\
\hline $\mathrm{sS} / \mathrm{T}$ & $1.60 \pm 0.30$ & $1.4-1.8$ & 1.6 & $1.50 \pm 0.30$ & $1.3,1.7$ & 1.4 & -0.1 & 0.147 \\
\hline \multicolumn{9}{|c|}{ Biochemical parameters } \\
\hline TC & $4.7 \pm 0.8$ & $4.3,5.1$ & 4.7 & $4.5 \pm 1.0$ & $4.0,5.1$ & 4.4 & -0.2 & 0.82 \\
\hline LDL-C & $2.7 \pm 0.7$ & $2.3,3.0$ & 2.7 & $2.4 \pm 0.6$ & $2.1,2.8$ & 2.5 & -0.2 & 0.198 \\
\hline HDL-C & $1.5 \pm 0.4$ & $1.3,1.7$ & 1.4 & $1.6 \pm 0.5$ & $1.3,1.9$ & 1.4 & 0.1 & 0.173 \\
\hline TAG & $1.4 \pm 1.3$ & $0.7,2.1$ & 0.8 & $1.5 \pm 1.8$ & $0.5,2.5$ & 1.1 & 0.1 & 0.691 \\
\hline $\mathrm{TP}$ & $69.4 \pm 5.2$ & $66.4,72.5$ & 69.8 & $73 \pm 4.6$ & $70.4,75.5$ & 73.6 & 3.5 & $0.026^{*}$ \\
\hline $\mathrm{HbA}_{1 \mathrm{C}} \% \mathrm{NGSP}$ & $8.8 \pm 3.7$ & $7.9,9.7$ & 8.3 & $8.3 \pm 3.3$ & $7.6,9.0$ & 8.0 & -2.7 & 0.362 \\
\hline $\mathrm{HbA}_{1 \mathrm{C}} \mathrm{mmol} / \mathrm{mol}$ & $73 \pm 17$ & 63,82 & 67.0 & $67 \pm 13$ & 60,75 & 64.0 & -6 & 0.362 \\
\hline TDDI & $55.6 \pm 17.7$ & $45.8,65.4$ & 55.0 & $44.8 \pm 10.4$ & $39.1,50.6$ & 42.0 & -10.8 & $0.002^{* *}$ \\
\hline I.U./kg & $0.67 \pm 0.17$ & $0.57,0.76$ & 0.69 & $0.53 \pm 0.11$ & $0.47,0.59$ & 0.51 & -0.14 & $0.002^{* *}$ \\
\hline \multicolumn{9}{|c|}{ Hormonal parameters } \\
\hline Leptin & $10,427 \pm 14,137$ & $3,273,17,581$ & 5,523 & $6,661 \pm 9,042$ & $1,925,11,398$ & 2,740 & $-4,116$ & 0.3 \\
\hline Ghrelin & $2,912 \pm 481$ & $2,668,3,155$ & 3,105 & $2,713 \pm 858$ & $2,263,3,162$ & 2,541 & -256 & 0.3 \\
\hline \multicolumn{9}{|c|}{ Glycaemic variability } \\
\hline SD & $4.2 \pm 0.6$ & $3.9,4.5$ & 4.3 & $3.3 \pm 0.5$ & $3.1,3.6$ & 3.3 & -0.8 & $0.001^{* *}$ \\
\hline ADRR & $18.4 \pm 1.6$ & $17.6,19.3$ & 18.2 & $17.4 \pm 0.8$ & $16.9,17.8$ & 17.1 & -1.0 & $0.006^{* *}$ \\
\hline \multicolumn{9}{|l|}{ Energy expenditure } \\
\hline METs/w & $225.6 \pm 18.8$ & $215.2,236$ & 231 & $233.9 \pm 17.8$ & $224,243.7$ & 235.1 & 9.0 & $0.005^{* *}$ \\
\hline $\mathrm{Kcal} / \mathrm{kg} /$ day & $32.2 \pm 2.7$ & $30.7,33.7$ & 33 & $33.4 \pm 2.6$ & $32,34.8$ & 33.6 & 1.3 & $0.005^{* *}$ \\
\hline
\end{tabular}

BW, body weight $(\mathrm{kg})$; BMI, body mass index $\left(\mathrm{kg} / \mathrm{m}^{2}\right)$; WC, waist circumference $(\mathrm{cm})$; NC, neck circumference ( $\left.\mathrm{cm}\right)$; TBF, total body fat (\%); VFA, visceral fat area $\left(\mathrm{cm}^{2}\right)$; SMM, skeletal muscle mass (kg); BS, biceps skinfold; TS, triceps skinfold; sSS, subscapular skinfold, SIS, supra-iliac skinfold; sSS+SIS, subscapular+supra-iliac skinfold sum (cm); sS/T, subscapular-to-triceps ratio; TC, total cholesterol (mmol/L); LDL-C, low-density lipoprotein cholesterol (mmol/L); HDL-C, high-density lipoprotein cholesterol (mmol/L); TAG, triglycerides (mmol/L); TP, total proteins (g/L), HbA1c, glycated haemoglobin; TDDI, total daily dose insulin; I.U./kg, unit of insulin/ kg body weight; leptin, in pg/mL; ghrelin, in pg/mL; SD, standard deviation; ADRR, average daily risk range; METs/w physical activity energy expenditure, metabolic equivalents/weekly; kcal/kg/day, kilocalories/kilogram/day; MV, mean value; 95\% CI, confidence interval; Med(x), median; * $\mathrm{p}$ value (Wilcoxon signed rank test) $<0.05$ is statistically significant, ** $\mathrm{p}$ value (Wilcoxon signed rank test) $<0.01$. 
Prídavková et al.: Insulin Pump Therapy - Influence on Body Fat Redistribution, Skeletal Muscle Mass and Ghrelin, Leptin Changes in T1D Patients

Table 2. Anthropometric, biochemical parameters, glycaemic variability, energy expenditure in women

\begin{tabular}{|c|c|c|c|c|c|c|c|c|}
\hline \multirow{2}{*}{$\begin{array}{l}\text { Women } \\
n=16\end{array}$} & \multicolumn{3}{|l|}{ Before pump } & \multicolumn{3}{|l|}{ On pump } & \multirow{2}{*}{$\begin{array}{l}\text { Mean } \\
\text { change }\end{array}$} & \multirow[t]{2}{*}{$p$ value } \\
\hline & $\mathrm{MV} \pm \mathrm{SD}$ & $95 \% \mathrm{CI}$ & Med (x) & $\mathrm{MV} \pm \mathrm{SD}$ & $95 \% \mathrm{CI}$ & Med (x) & & \\
\hline \multicolumn{9}{|c|}{ Anthropometric parameters } \\
\hline BW & $66.8 \pm 11.1$ & $60.9,72.8$ & 67.0 & $66.6 \pm 10.6$ & $61.0,72.3$ & 67.0 & -0.2 & 0.968 \\
\hline BMI & $24.1 \pm 3.1$ & $22.5,25.8$ & 24.6 & $24.1 \pm 3.0$ & $22.5,25.6$ & 23.6 & -0.1 & 0.937 \\
\hline WC & $78.6 \pm 12.9$ & $71.7,85.5$ & 73.5 & $77.6 \pm 11.1$ & $71.7,83.5$ & 73.0 & -1.0 & 0.724 \\
\hline NC & $33.2 \pm 2.5$ & $31.8,34.5$ & 32.8 & $33.1 \pm 2.2$ & $31.9,34.3$ & 33.0 & -0.03 & 0.917 \\
\hline \multicolumn{9}{|c|}{ Bioelectrical impedance analysis } \\
\hline TBF & $26.6 \pm 8.0$ & $22.3,30.8$ & 27.9 & $22.6 \pm 7.5$ & $18.6,26.6$ & 23.0 & -4.0 & $0.020^{*}$ \\
\hline VFA & $57.2 \pm 29.5$ & $41.5,72.8$ & 65.9 & $50.0 \pm 28.4$ & $34.8,65.1$ & 45.3 & -7.2 & 0.098 \\
\hline SMM & $27.0 \pm 4.9$ & $24.4,29.6$ & 25.2 & $28.8 \pm 5.3$ & $26.0,31.6$ & 28.9 & 1.8 & $0.008^{* *}$ \\
\hline \multicolumn{9}{|l|}{ Skinfold thickness } \\
\hline BS & $1.15 \pm 0.54$ & $0.86,1.44$ & 1.05 & $1.11 \pm 0.47$ & $0.86,1.37$ & 1.0 & -0.04 & 0.774 \\
\hline TS & $2.29 \pm 0.81$ & $1.86,2.72$ & 2.1 & $2.27 \pm 0.77$ & $1.86,2.68$ & 2.15 & -0.03 & 0.897 \\
\hline sSS & $1.81 \pm 0.53$ & $1.53,2.09$ & 1.85 & $1.81 \pm 0.38$ & $1.61,2.01$ & 1.9 & 0.0 & 0.85 \\
\hline SIS & $2.03 \pm 0.68$ & $1.67,2.39$ & 2.18 & $2.17 \pm 0.62$ & $1.84,2.50$ & 2.3 & 2.14 & 0.305 \\
\hline sSS+SIS & $3.84 \pm 1.04$ & $3.29,4.39$ & 3.93 & $3.98 \pm 0.78$ & $3.56,4.39$ & 4.3 & 0.14 & 0.393 \\
\hline sS/T & $0.9 \pm 0.4$ & $0.70,1.10$ & 0.8 & $0.90 \pm 0.40$ & $0.70,1.10$ & 0.8 & 0.0 & 0.469 \\
\hline \multicolumn{9}{|c|}{ Biochemical parameters } \\
\hline TC & $4.7 \pm 0.9$ & $4.2,5.2$ & 4.6 & $4.8 \pm 0.8$ & $4.4,5.3$ & 4.8 & 0.1 & 0.642 \\
\hline LDL-C & $2.7 \pm 0.9$ & $2.2,3.2$ & 2.8 & $2.6 \pm 0.7$ & $2.2,3.0$ & 2.5 & -0.1 & 0.532 \\
\hline HDL-C & $1.5 \pm 0.4$ & $1.3,1.7$ & 1.4 & $1.9 \pm 0.4$ & $1.6,2.1$ & 1.8 & 0.4 & $0.002^{* *}$ \\
\hline TAG & $1.0 \pm 0.5$ & $0.8,1.3$ & 0.9 & $0.9 \pm 0.4$ & $0.7,1.1$ & 0.8 & -0.1 & 0.14 \\
\hline $\mathrm{TP}$ & $65.3 \pm 4.3$ & $62.9,67.7$ & 65.9 & $73.1 \pm 4.1$ & $70.8,75.3$ & 72.7 & 7.8 & $0.001^{* *}$ \\
\hline $\mathrm{HbA}_{1 \mathrm{c}} \% \mathrm{NGSP}$ & $9.4 \pm 4.0$ & $8.4,10.4$ & 9.3 & $8.0 \pm 3.1$ & $7.5,8.6$ & 7.9 & -3.4 & $0.008^{* *}$ \\
\hline $\mathrm{HbA}_{1 \mathrm{c}} \mathrm{mmol} / \mathrm{mol}$ & $79 \pm 20$ & 68,90 & 78 & $64 \pm 10$ & 59,70 & 63 & -14 & $0.008^{* *}$ \\
\hline TDDI & $39.3 \pm 12.3$ & $32.8,45.9$ & 36.0 & $30.7 \pm 8.0$ & $26.4,35.0$ & 30.0 & -8.6 & $0.001^{* *}$ \\
\hline I.U./kg & $0.59 \pm 0.16$ & $0.51,0.68$ & 0.61 & $0.47 \pm 0.12$ & $0.40,0.53$ & 0.49 & -0.13 & $0.001^{* *}$ \\
\hline \multicolumn{9}{|c|}{ Hormonal parameters } \\
\hline Leptin & $15,392 \pm 15,823$ & $7,639,23,145$ & 10,800 & $4,985 \pm 3,960$ & $3,044,6,925$ & 3,751 & $-10,407$ & $0.010^{*}$ \\
\hline Ghrelin & $3,146 \pm 952$ & $2,680,3,613$ & 2,894 & $2,503 \pm 1,060$ & $1,984,3,023$ & 2,132 & -643 & $0.044^{*}$ \\
\hline \multicolumn{9}{|c|}{ Glycaemic variability } \\
\hline SD & $4.2 \pm 0.5$ & $4.0,4.5$ & 4.3 & $3.2 \pm 0.4$ & $3.0,3.4$ & 3.2 & -1.1 & $<0.001^{* * *}$ \\
\hline ADRR & $20.8 \pm 5.6$ & $17.9,23.8$ & 19.2 & $17 \pm 1.0$ & $16.5,17.5$ & 17.2 & -2.2 & $0.001^{* *}$ \\
\hline \multicolumn{9}{|c|}{ Energy expenditure } \\
\hline METs/w & $209.2 \pm 23.4$ & $196.7,221.7$ & 203 & $220.8 \pm 19.4$ & $210.4,231.2$ & 219 & 14 & $0.003^{* *}$ \\
\hline Kcal/kg/day & $29.7 \pm 3.2$ & $28.0,31.4$ & 29 & $31.3 \pm 4.2$ & $29.1,33.6$ & 31.3 & 1.9 & $0.048^{*}$ \\
\hline
\end{tabular}

BW, body weight $(\mathrm{kg})$; BMI, body mass index $\left(\mathrm{kg} / \mathrm{m}^{2}\right)$; WC, waist circumference $(\mathrm{cm})$; NC, neck circumference (cm); TBF, total body fat (\%); VFA, visceral fat area $\left(\mathrm{cm}^{2}\right)$; SMM, skeletal muscle mass (kg); BS, biceps skinfold; TS, triceps skinfold; sSS, subscapular skinfold; SIS, supra-iliac skinfold; sSS+SIS, subscapular+supra-iliac skinfold sum (cm); sS/T, subscapular to triceps ratio; TC, total cholesterol (mmol/L), LDL-C, low-density lipoprotein cholesterol (mmol/L); HDL-C, high-density lipoprotein cholesterol (mmol/L); TAG, triglycerides (mmol/L); TP, total proteins (g/L), $\mathrm{HbA}_{1 \mathrm{c}}$, glycated haemoglobin; TDDI, total daily dose insulin; I.U./kg, unit of insulin/kg body weight; leptin, in $\mathrm{pg} / \mathrm{mL}$; ghrelin, in $\mathrm{pg} / \mathrm{mL}$; SD, standard deviation; ADRR, average daily risk range; METs/w physical activity energy expenditure, metabolic equivalents/weekly; kcal/kg/day, kilocalories/kilogram/day; MV, mean value; $95 \% \mathrm{CI}$, confidence interval; $\operatorname{Med}(\mathrm{x})$, median; ${ }^{*} p$ value (Wilcoxon signed rank test) $<0.05$ is statistically significant, ${ }^{* *} p$ value (Wilcoxon signed rank test) $<0.01$. 
Prídavková et al.: Insulin Pump Therapy - Influence on Body Fat Redistribution, Skeletal Muscle Mass and Ghrelin, Leptin Changes in T1D Patients

Table 3. The correlation between the changes in anthropometric and biochemical parameters

\begin{tabular}{|c|c|c|c|c|c|c|c|c|c|c|c|}
\hline & & TBF & VFA & SMM & sSS & sSS+SIS & TS & BS & $\mathrm{sS} / \mathrm{T}$ & TAG & HDL-C \\
\hline \multirow[t]{2}{*}{ BMI } & M & $0.006^{* *}$ & NS & NS & $0.008^{* *}$ & $0.005^{* *}$ & $0.005^{* *}$ & NS & $-0.043^{*}$ & NS & NS \\
\hline & W & NS & NS & NS & NS & NS & NS & NS & NS & NS & NS \\
\hline \multirow[t]{2}{*}{ BW } & M & $0.005^{* *}$ & NS & NS & $0.010^{*}$ & $0.005^{* *}$ & $0.008^{* *}$ & NS & NS & NS & NS \\
\hline & W & NS & NS & NS & NS & NS & NS & NS & NS & NS & NS \\
\hline \multirow[t]{2}{*}{ WC } & M & $0.001^{* *}$ & NS & NS & $0.042^{*}$ & $0.005^{* *}$ & NS & NS & NS & NS & NS \\
\hline & W & NS & NS & NS & $0.034^{*}$ & NS & NS & $0.013^{*}$ & NS & NS & NS \\
\hline \multirow[t]{2}{*}{ NC } & M & NS & NS & NS & NS & NS & NS & NS & NS & NS & NS \\
\hline & W & NS & NS & NS & $0.002^{* *}$ & NS & $0.003^{* *}$ & NS & NS & NS & NS \\
\hline \multirow[t]{2}{*}{ VFA } & M & $0.027^{*}$ & - & $-0.001^{* *}$ & $0.014^{*}$ & 0.038* & NS & NS & NS & NS & NS \\
\hline & W & $0.043^{*}$ & - & NS & NS & NS & NS & NS & NS & NS & $-0.015^{*}$ \\
\hline \multirow[t]{2}{*}{ TBF } & M & - & $0.027^{*}$ & $0.016^{*}$ & $0.001^{* *}$ & 0.018* & NS & NS & NS & NS & NS \\
\hline & W & - & $0.043^{*}$ & $-0.005^{* *}$ & NS & $0.022^{*}$ & NS & NS & NS & $-0.009^{* *}$ & NS \\
\hline \multirow[t]{2}{*}{ Leptin } & M & NS & NS & NS & NS & NS & NS & NS & NS & NS & NS \\
\hline & W & $0.024^{*}$ & NS & $-0.024^{*}$ & NS & NS & NS & NS & NS & NS & NS \\
\hline \multirow[t]{2}{*}{ Ghrelin } & M & NS & NS & NS & NS & NS & NS & NS & NS & NS & NS \\
\hline & W & NS & NS & NS & NS & NS & NS & NS & NS & NS & $-0.035^{*}$ \\
\hline \multirow[t]{2}{*}{ HDL-C } & M & NS & NS & NS & NS & NS & $-0.018^{*}$ & NS & NS & NS & - \\
\hline & W & NS & $-0.015^{*}$ & NS & NS & NS & NS & NS & NS & NS & - \\
\hline
\end{tabular}

BMI, body mass index; BW, body weight; WC, waist circumference; NC, neck circumference; VFA, visceral fat area; TBF, total body fat; HDL-C, high-density lipoprotein cholesterol; SMM, skeletal muscle mass; sSS, subscapular skinfold; sSS + SIS, subscapular + supra-iliac skinfolds sum; TS, triceps skinfold; BS, biceps skinfold; sS/T, subscapular-to-triceps ratio; TAG, triglycerides; $p$ value, $<0.05^{*}$ is statistically significant; NS, not significant.

Fig. 1. Mean changes (\%) before pump therapy and on pump therapy. BMI, body mass index; BW, body weight; WC, waist circumference; NC, neck circumference; VFA, visceral fat area; TBF, total body fat; SMM, skeletal muscle mass; $p$ value, $<0.05^{*}$ is statistically significant.

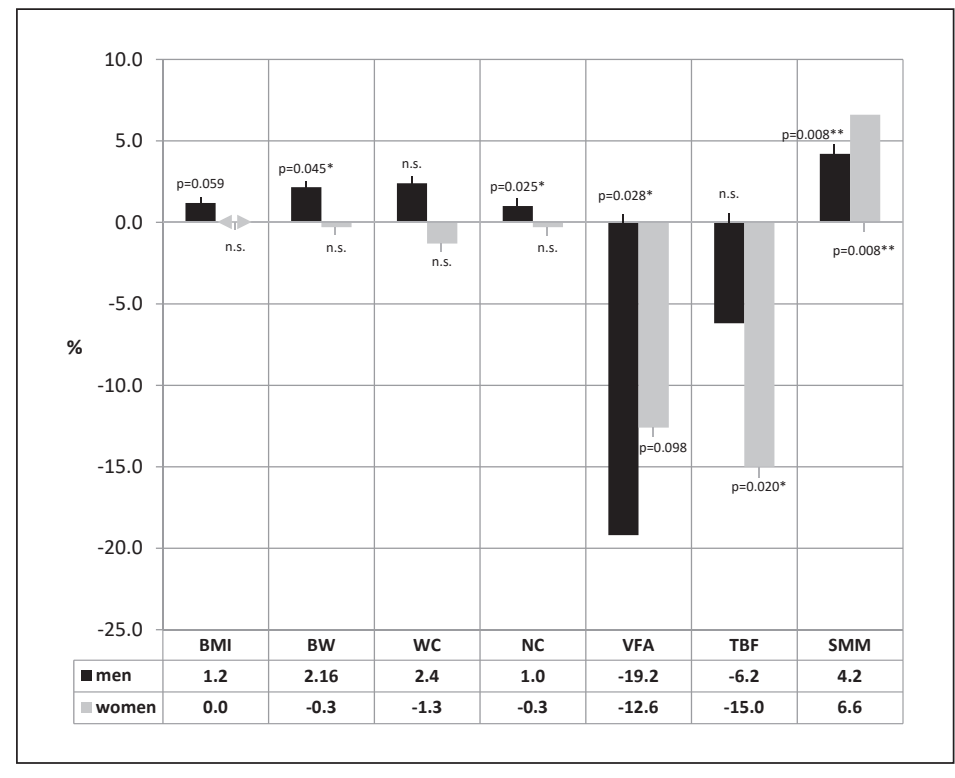

Fig. 1). In women, we found inverse correlation between SMM and TBF ( $p=-0.005)$, and in men between SMM and VFA ( $p=-0.001)$ (Table 3). The levels of leptin decreased significantly in women only ( $p=0.010)$ (Table 2, Fig. 2$)$; these correlated with TBF ( $p=0.024)$ and inversely with SMM ( $p=-0.024)$, but we did not prove correlation in men (Table 3). BW increased in men only ( $p=0.045)$ (Table 1 , Fig. 1$)$ and correlated with TBF $(p=0.005)$, $\operatorname{SSS}+\operatorname{SIS}(p=0.005)$ 
Prídavková et al.: Insulin Pump Therapy - Influence on Body Fat Redistribution, Skeletal Muscle Mass and Ghrelin, Leptin Changes in T1D Patients

Table 4. The correlation between the changes in anthropometric parameters and glycaemic variability, physical activity, ghrelin in men and women

\begin{tabular}{|c|c|c|c|c|c|c|c|}
\hline & TBF & VFA & SMM & BW & sSS & SIS & Ghrelin \\
\hline \multicolumn{8}{|l|}{ ADRR } \\
\hline Men & $-0.026^{*}$ & NS & NS & NS & NS & NS & NS \\
\hline Women & NS & NS & NS & NS & NS & NS & NS \\
\hline \multicolumn{8}{|l|}{ SD } \\
\hline Men & NS & NS & NS & NS & NS & NS & NS \\
\hline Women & NS & NS & NS & NS & NS & NS & NS \\
\hline \multicolumn{8}{|l|}{ METs/w } \\
\hline Men & NS & $-0.002^{* *}$ & $0.008^{* *}$ & NS & NS & NS & $-0.040^{*}$ \\
\hline Women & $-0.001^{* *}$ & $-0.006^{* *}$ & NS & $-0.028^{*}$ & $-0.007^{* *}$ & $-0.016^{*}$ & NS \\
\hline
\end{tabular}

TBF, total body fat; VFA, visceral fat area; SMM, skeletal muscle mass; BW, body weight; sSS, subscapular skinfold; supra-iliacal skinfold; ADRR, average daily risk range; SD, standard deviation (glycaemic variability); METs/w, metabolic equivalents/weekly; $p$ value, $p<0.05^{*}$ is statistically significant; NS, not significant.

and TS ( $p=0.008$ ) (Table 3). We found an inverse correlation between ghrelin levels and HDL-C ( $p=-0.035)$ in women (Table 3$)$ and an inverse correlation between ghrelin levels and METs ( $p=-0.040)$ in men (Table 4$)$. In men, physical activity (METs/w) increased ( $p=0.005)$ and correlated negatively with VFA $(p=-0.002)$. In women, physical activity increased ( $p=$ $0.003)$ and correlated negatively with VFA $(p=-0.006)$ (Table 4$)$.

\section{Discussion}

At one and the same BMI, women typically present with $10 \%$ higher body fat compared to men, and in general women are more effective in storing fat subcutaneously and men intraabdominally [21]. Women in our group had higher TBF, as shown by greater thickness of subcutaneous fat and higher visceral fat mass, when compared to males. Higher visceral fat tissues in females with DM has also been reported by Ye et al. [4]. Higher VFA in women with T1D could be related to more frequent oscillations in glycaemia during the menstrual cycle. Counterregulatory hormones, especially cortisol, are activated in hypoglycaemia, which adds to the increase of visceral fat, and there are more glu cocorticoid receptors compared to subcutaneous adipocytes [22]. The flexible regimen of insulin pump use during physical activity most likely added to visceral fat mass reduction in both sexes as we observed in our group. Much like in our group, a higher level of physical activity was correlated negatively with the VFA changes in patients with T1D [10], but was also assessed in patients without diabetes [23].

Sex is an important factor for different body fat distribution. It has been reported that testosterone induces meal free fatty acid (FFA) uptake preferentially into abdominal subcutaneous adipose tissue (SAT) resulting in a decreased uptake in visceral adipose tissue (VAT) [24]. The biological effect of testosterone is most likely enhanced by decreasing leptin levels, and an inverse correlation between leptin levels and testosterone was reported by Kelsey et al. [25]. Even though we did not measure testosterone levels, we assume that decreasing leptin levels contributed to a decrease of visceral fat and an increase of subcutaneous skinfold thickness in the trunk area induced by testosterone. We confirmed that VFA inversely correlated with HDL-C in females, but not in men. In women, there was a significant increase of HDL-C on insulin pump. This was not observed in men, most likely due to higher smoker 
Prídavková et al.: Insulin Pump Therapy - Influence on Body Fat Redistribution, Skeletal Muscle Mass and Ghrelin, Leptin Changes in T1D Patients

representation, but also because of worse glycaemic compensation (evaluated by HbA1c) when compared to women. The increase of HDL-C after switch on intensified insulin treatment in T1D patients was also observed by other authors [26-28] and recently also in T2D patients as shown in the 0pT2mise study [29].

More studies have reported a BW increase in T1D patients when starting insulin treatment and several mechanisms have been suggested; primary one is decreased urinary caloric loss, another cause is the reversal of the metabolic state, from catabolism to anabolism, which is mediated by insulin [7]. The effect of insulin on proteins, leading to an increase in lean body mass [30] was seen in both men and women after switching from MDI to CSII. We presume that the SMM increase on CSII treatment, rather than MDI treatment, is due to the combination of higher physical activity, the lower catabolic effect of cortisol, and a metabolically more effective protein anabolic effect of insulin. A BMI increase was found in men only (Table 1, Fig. 1). In DCCT study, the BMI increase in women was higher than in men; however, the additional weight includes lean tissue as well as fat [31,32]. A possible explanation for this difference may be a higher age spectrum in the DCCT group with together with a longer duration of the study. In our study, there was an increase of BW in men only, although Holl et al. [33] postulated that, along with high insulin doses, female gender predisposes to weight gain. Studies in T1D patients comparing the MDI regimen to CSII found no significant differences with regard to BW $[34,35]$.

Skinfold thickness, much like NC and WC, had increased in men; however, significant changes were only found in supra-iliac skinfold and NC. After starting insulin therapy in T2D, Takei at al. [36] observed a similar trend, i.e., a significant decrease in the ratio of visceral-tosubcutaneous fat area, this was mainly due to an increase in the subcutaneous fat amounts. The changes of skinfold thicknesses in upper body in women, were not statistically significant, which is most likely related to physiologically lower activity of lipoprotein lipase (LPL) adipocytes in this area [37] as per oestrogen effect, compared to men, in which LPL adipocyte activity in abdominal SAT is higher [24]. Different levels of lipolysis may be mediated, in part, through a higher expression of lipolytic beta-adrenergic receptors in the upper body depot [24]. It appears that skinfold thickness assessment provides helpful indicators for an adverse lipid profile in paediatric population also [38,39].

One of the main actions of exogenous ghrelin is to stimulate appetite [40]. Ghrelin levels were significantly decreased in women, but not in men, and did not inversely correlate with either BMI or VFA as reported by another T1D study [10]. In men, ghrelin levels correlated inversely with physical activity, and similar data are provided by Ozen-Vatansever et al. [41], even though they did not observe patients with T1D. Reduced ghrelin concentration after acute moderate intensity exercise might be due to increased sympathetic nervous system activity and decreased perfusion of gastric mucosa resulting from redistribution of blood flow from the splanchnic circulation towards the skeletal muscles during exercise [41]. The effects of exercise on ghrelin are rather controversial and depend on the intensity and duration of exercise $[42,43]$.

Likewise, in patients with diabetes it appears that ghrelin is not the main factor controlling appetite. We did not find a correlation between ghrelin and HbA1c, which can be explained by ghrelin levels being influenced more by acute than chronic changes in plasma glucose concentration.

Leptin, an adipocyte-secreted hormone, was shown to be predominantly expressed in isolated subcutaneous adipocytes as opposed to omental adipocytes, particularly in women [24]. In our group, leptin decreased significantly in women, which is most likely related to a higher initial level, and to a higher loss of total body fat (Table 2, Fig. 1, 2). A decrease in the leptin levels in newly diagnosed T1D patients and an increase of insulin therapy was reported by Sariano-Guillén et al. [40] where this increase was independent of changes in BW and was 
Fig. 2. Changes of leptin and ghrelin before and on pump therapy. $\mathrm{BP}$, before pump; OP, on pump; $* p$ value, $<0.05$ is statistically significant.

Prídavková et al.: Insulin Pump Therapy - Influence on Body Fat Redistribution, Skeletal Muscle Mass and Ghrelin, Leptin Changes in T1D Patients

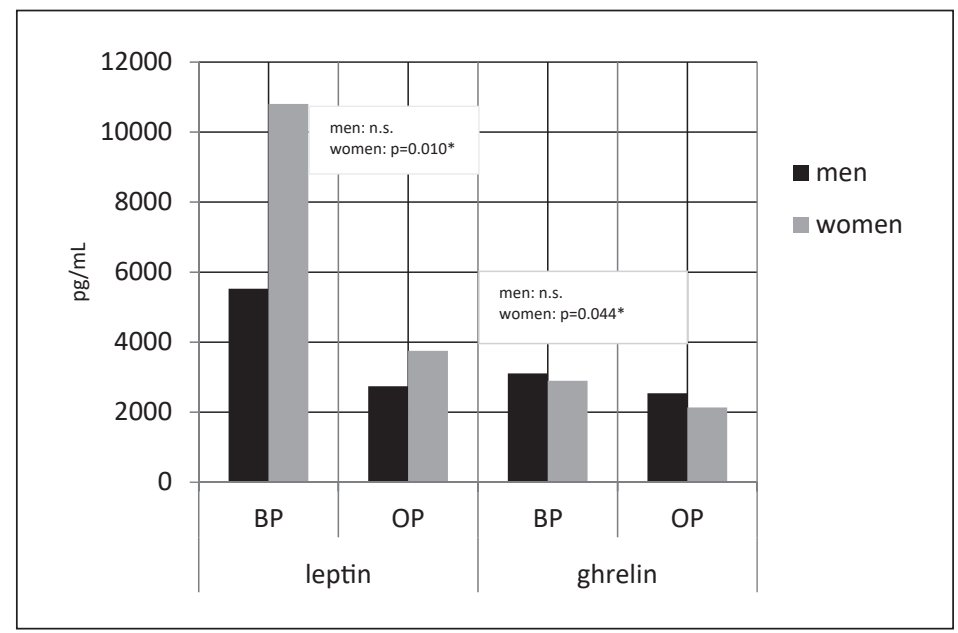

probably due to the stimulatory effect of insulin on leptin production. While leptin and ghrelin levels showed a decreasing tendency, they are thought to exert opposite effects on metabolism [40].

To summarise, the study presents the positive influence CSII on the body fat distribution and proteoanabolism (decreased visceral fat, increased skeletal muscle mass and plasma proteins). It showed that this state was influenced by increase of physical activity and partially by improved GV, although, to prove this, more patients would have been necessary. It appears that the changes of BW do not reflect the changes in fat and lean mass correctly, and more sophisticated methods for body composition evaluation are needed. Finally, a few limitations are worth noting: First, the present study has only a small sample size; a much larger study will need to be done in order to confirm some of these findings. Second, more sophisticated methods for body composition evaluation would be dual X-ray densitometry, computed tomography or magnetic resonance imaging. Third, physical activity was evaluated by the 7-Day Physical Activity Recall, although pedometers or accelerometers and pulse rate assessments are currently considered the only methods providing sophisticated data on physical activity. Furthermore, due to the small sample size we were unable to analyse the changes in body composition and body fat distribution in relation to age, sex, obesity class and GV.

\section{Acknowledgement}

This work was supported by project 'The increasing of opportunities for career growth in research and development in the medical sciences', co-financed from EU sources, ITMS: 26110230067.

\section{Author Disclosure Statement}

The authors have nothing to disclose. 
Prídavková et al.: Insulin Pump Therapy - Influence on Body Fat Redistribution, Skeletal Muscle Mass and Ghrelin, Leptin Changes in T1D Patients

\section{References}

1 Momesso DP, Bussade I, Lima GA, Fonseca LP, Russo LA, Kupfer R. Body composition, metabolic syndrome and insulin resistance in type 1 diabetes mellitus. Arq Bras Endocrinol Metabol. 2011 Apr;55(3):189-93.

2 Łuczyński W, Szypowska A, Głowińska-Olszewska B, Bossowski A. Overweight, obesity and features of metabolic syndrome in children with diabetes treated with insulin pump therapy. Eur J Pediatr. 2011 Jul;170(7): 891-8.

3 Gallagher D, Kelley DE, Yim JE, Spence N, Albu J, Boxt L, et al.; MRI Ancillary Study Group of the Look AHEAD Research Group. Adipose tissue distribution is different in type 2 diabetes. Am J Clin Nutr. 2009 Mar;89(3): 807-14.

4 Ye Y, Bao Y, Hou X, Pan X, Wu H, Li H, et al. Identification of waist circumference cutoffs for abdominal obesity in the Chinese population: a 7.8-year follow-up study in the Shanghai urban area. Int J Obes. 2009 Sep;33(9): 1058-62.

5 Nathan DM, Genuth S, Lachin J, Cleary P, Crofford O, Davis M, et al.; Diabetes Control and Complications Trial Research Group. The effect of intensive treatment of diabetes on the development and progression of longterm complications in insulin-dependent diabetes mellitus. N Engl J Med. 1993 Sep;329(14):977-86.

6 The DCCT Research Group. Weight gain associated with intensive therapy in the diabetes control and complications trial. Diabetes Care. 1988 Jul-Aug;11:567-73.

7 Jacob AN, Salinas K, Adams-Huet B, Raskin P. Potential causes of weight gain in type 1 diabetes mellitus. Diabetes Obes Metab. 2006 Jul;8(4):404-11.

8 Lipsky LM, Gee B, Liu A, Nansel TR. Glycemic control and variability in association with body mass index and body composition over 18 months in youth with type 1 diabetes. Diabetes Res Clin Pract. 2016 Oct;120: 97-103.

9 Boucher-Berry C, Parton EA, Alemzadeh R. Excess weight gain during insulin pump therapy is associated with higher basal insulin doses. J Diabetes Metab Disord. 2016 Oct;15(1):47.

10 Jacob AN, Adams-Huet B, Raskin P. The visceral and subcutaneous fat changes in type 1 diabetes: a pilot study. Diabetes Obes Metab. 2006 Sep;8(5):524-30.

11 The Diabetes Control And Complications Trial Research Group. Influence of intensive diabetes treatment on body weight and composition of adults with type 1 diabetes in the Diabetes Control and Complications Trial. Diabetes Care. 2001 Oct;24(10):1711-21.

12 World Health Organization. Obesity: Preventing and Managing the Global Epidemic. Report of a WHO Consultation (WHO Technical Report Series 894), World Health Organization;2000. http://www.who.int/nutrition/ publications/obesity/WHO_TRS_894/en/

13 Waist Circumference and Waist-Hip Ratio. Report of a WHO expert consultation, Geneva; 2008. http://apps. who.int/iris/bitstream/handle/10665/44583/?sequence=1

14 Zen V, Fuchs FD, Wainstein MV, Gonçalves SC, Biavatti K, Riedner CE, et al. Neck circumference and central obesity are independent predictors of coronary artery disease in patients undergoing coronary angiography. Am J Cardiovasc Dis. 2012;2(4):323-30.

15 Marfell-Jones M, Olds T, Stewart A, Carter L. International Standards for Anthropometric Assessment. Potchefstroom, International Society for the Advancement of Kinathropometry (ISAK), 2006, pp 1-137.

16 Kovatchev BP, Otto E, Cox D, Gonder-Frederick L, Clarke W. Evaluation of a new measure of blood glucose variability in diabetes. Diabetes Care. 2006 Nov;29(11):2433-8.

17 Siegelaar SE, Holleman F, Hoekstra JB, DeVries JH. Glucose variability; does it matter? Endocr Rev. 2010 Apr; 31(2):171-82.

18 Sallis JF, Haskell WL, Wood PD, Fortmann SP, Rogers T, Blair SN, et al. Physical activity assessment methodology in the Five-City Project. Am J Epidemiol. 1985 Jan;121(1):91-106.

19 Ainsworth BE, Haskell WL, Herrmann SD, Meckes N, Bassett DR Jr, Tudor-Locke C, et al. 2011 Compendium of Physical Activities: a second update of codes and MET values. Med Sci Sports Exerc. 2011 Aug;43(8):1575-81.

20 Fletcher GF, Ades PA, Kligfield P, Arena R, Balady GJ, Bittner VA, et al.; American Heart Association Exercise, Cardiac Rehabilitation, and Prevention Committee of the Council on Clinical Cardiology, Council on Nutrition, Physical Activity and Metabolism, Council on Cardiovascular and Stroke Nursing, and Council on Epidemiology and Prevention. Exercise standards for testing and training: a scientific statement from the American Heart Association. Circulation. 2013 Aug;128(8):873-934.

21 Karastergiou K, Smith SR, Greenberg AS, Fried SK: Sex differences in human adipose tissues - the biology of pear shape. Biol Sex Differ. 2012;3(1):13.

22 Dallman MF, la Fleur SE, Pecoraro NC, Gomez F, Houshyar H, Akana SF. Minireview: glucocorticoids-food intake, abdominal obesity, and wealthy nations in 2004. Endocrinology. 2004 Jun;145(6):2633-8.

23 Ross R, Janiszewski PM. Is weight loss the optimal target for obesity-related cardiovascular disease risk reduction? Can J Cardiol. 2008;24(Suppl D):25D-31D.

24 White UA, Tchoukalová YD. Sex dimorphism and depot differences in adipose tissue function. Biochim Biophys Acta. 2014 Mar;1842(3):377-92.

25 Kelsey MM, Bjornstad P, McFann K, Nadeau K. Testosterone concentration and insulin sensitivity in young men with type 1 and type 2 diabetes. Pediatr Diabetes. 2016 May;17(3):184-90.

26 Wilson DP, Fesmire JD, Endres RK, Blackett PR. Increased levels of HDL-cholesterol and apolipoprotein A-I after intensified insulin therapy for diabetes. South Med J. 1985 Jun;78(6):636-8. 
Prídavková et al.: Insulin Pump Therapy - Influence on Body Fat Redistribution, Skeletal Muscle Mass and Ghrelin, Leptin Changes in T1D Patients

27 Kahri J, Groop PH, Viberti G, Elliott T, Taskinen MR. Regulation of apolipoprotein A-I-containing lipoproteins in IDDM. Diabetes. 1993 Sep;42(9):1281-8.

28 Fukui T, Hirano T. High-density lipoprotein subspecies between patients with type 1 diabetes and type 2 diabetes without / with intensive insulin therapy. Endocr J. 2012;59(7):561-9.

29 Reznik Y, Cohen O, Aronson R, Conget I, Runzis S, Castaneda J, et al.; OpT2mise Study Group. Insulin pump treatment compared with multiple daily injections for treatment of type 2 diabetes (OpT2mise): a randomised open-label controlled trial. Lancet. 2014 0ct;384(9950):1265-72.

30 Torbay N, Bracco EF, Geliebter A, Stewart IM, Hashim SA. Insulin increases body fat despite control of food intake and physical activity. Am J Physiol. 1985 Jan;248(1 Pt 2):R120-4.

31 The Diabetes Control and Complications Trial Research Group. Influence of intensive diabetes treatment on body weight and composition of adults with type 1 diabetes in the Diabetes Control and Complications Trial. Diabetes Care. 2001 Oct;24(10):1711-21.

32 Diabetes Control and Complications Trial Research Group. Effect of intensive diabetes treatment on the development and progression of long-term complications in adolescents with insulin-dependent diabetes mellitus: Diabetes Control and Complications Trial. J Pediatr. 1994 Aug;125(2):177-88.

33 Holl RW, Swift PG, Mortensen HB, Lynggaard H, Hougaard P, Aanstoot HJ, Chiarelli F, Daneman D, Danne T, Dorchy H, Garandeau P, Greene S, Hoey HM, Kaprio EA, Kocova M, Martul P, Matsuura N, Robertson KJ, Schoenle EJ, Sovik O, Tsou RM, Vanelli M, Aman J. Insulin injection regimens and metabolic control in an international survey of adolescents with type 1 diabetes over 3 years: results from the Hvidore study group. Eur J Pediatr. 2003 Jan;162(1):22-9.

34 Misso ML, Egberts KJ, Page M, O'Connor D, Shaw J. Continuous subcutaneous insulin infusion (CSII) versus multiple insulin injections for type 1 diabetes mellitus. Cochrane Database Syst Rev. 2010 Jan;(1):CD005103.

35 Gunn D, Mansell P. Glycaemic control and weight 7 years after Dose Adjustment For Normal Eating (DAFNE) structured education in Type 1 diabetes. Diabet Med. 2012 Jun;29(6):807-12.

36 Takei I, Takayama S, Yamauchi A, Nakamoto S, Kitamura Y, Katsukawa F, et al. Effect of insulin therapy on body fat distribution in NIDDM patients with secondary sulfonylurea failure: a preliminary report. Eur J Clin Nutr. 1998 Feb;52(2):153-4.

37 Tessier S, Riesco E, Lacaille M, Pérusse F, Weisnagel J, Doré J, et al. Impact of walking on adipose tissue lipoprotein lipase activity and expression in pre- and postmenopausal women. Obes Facts. 2010 Jun;3(3):191-9.

38 Maffeis C, Pietrobelli A, Grezzani A, Provera S, Tatò L. Waist circumference and cardiovascular risk factors in prepubertal children. Obes Res. 2001 Mar;9(3):179-87.

39 Morrison JA, Barton BA, Biro FM, Sprecher DL. The conjoint trait of low high-density lipoprotein cholesterol and high triglycerides in adolescent black and white males. Metabolism. 1998 May;47(5):514-21.

40 Soriano-Guillén L, Barrios V, Lechuga-Sancho A, Chowen JA, Argente J. Response of circulating ghrelin levels to insulin therapy in children with newly diagnosed type 1 diabetes mellitus. Pediatr Res. 2004 May;55(5): 830-5.

41 Vatansever-Ozen S, Tiryaki-Sonmez G, Bugdayci G, Ozen G. The effects of exercise on food intake and hunger: relationship with acylated ghrelin and leptin. J Sports Sci Med. 2011 Jun;10(2):283-91.

42 Hagobian TA, Sharoff CG, Stephens BR, Wade GN, Silva JE, Chipkin SR, et al. Effects of exercise on energy-regulating hormones and appetite in men and women. Am J Physiol Regul Integr Comp Physiol. 2009 Feb; 296(2):R233-42.

43 Shiiya T, Ueno H, Toshinai K, Kawagoe T, Naito S, Tobina T, et al. Significant lowering of plasma ghrelin but not des-acyl ghrelin in response to acute exercise in men. Endocr J. 2011;58(5):335-42. 